\title{
CHARACTERIZATIONS OF ALL-DERIVABLE POINTS IN NEST ALGEBRAS
}

\author{
JUN ZHU AND SHA ZHAO \\ (Communicated by Marius Junge)
}

\begin{abstract}
Let $\mathcal{A}$ be an operator algebra on a Hilbert space. We say that an element $G \in \mathcal{A}$ is an all-derivable point of $\mathcal{A}$ if every derivable linear mapping $\varphi$ at $G$ (i.e. $\varphi(S T)=\varphi(S) T+S \varphi(T)$ for any $S, T \in \operatorname{alg} \mathcal{N}$ with $S T=G$ ) is a derivation. Suppose that $\mathcal{N}$ is a nontrivial complete nest on a Hilbert space $H$. We show in this paper that $G \in a l g \mathcal{N}$ is an all-derivable point if and only if $G \neq 0$.
\end{abstract}

\section{INTRODUCTION AND PRELIMINARIES}

Let $\mathcal{H}$ and $\mathcal{K}$ be two Hilbert spaces. $B(H, K)$ stands for the set of all bounded linear operators from $H$ into $K$ and $B(H, H)$ is abbreviated to $B(H)$. Let $\mathcal{A}$ be an operator subalgebra in $B(H)$. We say that a linear mapping $\varphi$ from $\mathcal{A}$ into itself is a derivable mapping at $G$ if $\varphi(S T)=\varphi(S) T+S \varphi(T)$ for any $S, T \in \mathcal{A}$ with $S T=G$. We say that an element $G \in \mathcal{A}$ is an all-derivable point of $\mathcal{A}$ if every derivable linear mapping $\varphi$ at $G$ is a derivation. Let $N$ be a closed subspace in $H$. We use the symbol $I_{N}$ to denote the unit operator in $B(N)$. If $\mathcal{N}$ is a complete nest on $H$, then the nest algebra $\operatorname{alg} \mathcal{N}$ is the set of all operators that leave every member of $\mathcal{N}$ invariant.

As is well known, derivations are very important maps both in theory and applications. In general there are two directions in the study of the local actions of derivations of operator algebras. One is the well-known local derivation problem (see [9]). The other is to study conditions under which derivations of operator algebras can be completely determined by the action on some sets of operators. It is obvious that a linear map is a derivation if and only if it is derivable at all points. It is natural and interesting to ask the question whether or not a linear map is a derivation if it is derivable only at one given point.

We describe some of the results related to ours. Jing and Lu [7] showed that every derivable mapping $\varphi$ at 0 with $\varphi(I)=0$ on nest algebras is a derivation. Hou and Qi [6] showed that every idempotent with the range in $\mathcal{N}$ is an all-derivations in $\operatorname{alg} \mathcal{N}$, where $\mathcal{N}$ is a complete nest on Banach space. Li, Pan and $\mathrm{Xu}$ [11 proved that every derivable mapping $\varphi$ at 0 with $\varphi(I)=0$ on CSL algebras is a derivation. Zhu and Xiong in [16] showed that every element $G \in \mathcal{T} \mathcal{M}_{n}$ is an all-derivable

Received by the editors April 24, 2010 and, in revised form, January 6, 2011 and October 13, 2011.

2010 Mathematics Subject Classification. Primary 47L35, 47B47.

Key words and phrases. All-derivable point, nest algebra, derivable linear mapping at $G$.

This work is supported by the National Natural Science Foundation of China (No. 10771191). 
point of $\mathcal{T} \mathcal{M}_{n}$ if and only if $G \neq 0$, where $\mathcal{T} \mathcal{M}_{n}$ is the algebra of all $n \times n$ upper triangular matrices. For other relative references, see [1] $-[5],[8]-[10,[12]-15]$.

It is the aim of this paper to prove that an operator $G \in \operatorname{alg} \mathcal{N}$ is an all-derivable point of the nest algebra $\operatorname{alg} \mathcal{N}$ if and only if $G \neq 0$, where $\mathcal{N}$ is a nontrivial complete nest on a Hilbert space $H$ (i.e. there exists an element $N \in \mathcal{N}$ with $N \neq\{0\}, H$ ).

\section{All-Derivable points in Nest algebras}

Suppose that $N$ is a closed subspace in $H$. In this section, we always use the symbols $I_{N}$ to denote the unit operator on $N$. We easily prove the following two lemmas.

Lemma 2.1. Let $\mathcal{N}$ be a complete nest on a Hilbert space $H$. Then $G$ is an allderivation point of alg $\mathcal{N}$ if and only if $\lambda G$ is of alg $\mathcal{N}$ for any real number $\lambda \neq 0$.

Proof. $\varphi$ is a derivable mapping at $G \Leftrightarrow \varphi(S T)=\varphi(S) T+S \varphi(T)$ for any $S, T \in \mathcal{A}$ with $S T=G \Leftrightarrow \varphi(\lambda S T)=\varphi(\lambda S) T+\lambda S \varphi(T)$ for any $S, T \in \mathcal{A}$ with $S T=G \Leftrightarrow$ $\varphi(S T)=\varphi(S) T+S \varphi(T)$ for any $S, T \in \mathcal{A}$ with $S T=\lambda G$.

Lemma 2.2. Let $\mathcal{A}$ be an operator subalgebra with unit operator $I$ in $B(H)$, and let $\varphi$ be a linear mapping from $\mathcal{A}$ into itself. Suppose that $\varphi(X)=0$ for any invertible operator $X \in \mathcal{A}$. Then $\varphi \equiv 0$.

Proof. For arbitrary $X \in$ algN $\mathcal{N}$, there exists a real number $\lambda>\|X\|$. Then both $\lambda I-X$ and $2 \lambda I-X$ are two invertible operators. So $\varphi(\lambda I-X)=0$ and $\varphi(2 \lambda I-X)=$ 0 . It follows from the linearity of $\varphi$ that $\varphi(X)=0$.

The following is our main theorem in this paper.

Theorem 2.3. Let $\mathcal{N}$ be a nontrivial complete nest on a Hilbert space $H$. Then an operator $G \in$ alg $\mathcal{N}$ is an all-derivable point if and only if $G \neq 0$.

Proof. It is easy to check that 0 is not an all-derivable point since the identical mapping is a derivable linear mapping at the zero point on nest algebras, but it is not a derivation.

Suppose that $\varphi$ is a derivable linear mapping at $G \neq 0$ from alg $\mathcal{N}$ into itself. We only need to prove that $\varphi$ is a derivation. Let $N \in \mathcal{N}$ with $\{0\} \subset N \subset H$. Then all $2 \times 2$ operator matrices are always represented as relative to the orthogonal decomposition $H=N \oplus N^{\perp}$ in the proof of this theorem. Thus we may write

$$
G=\left[\begin{array}{cc}
D & E \\
0 & F
\end{array}\right]
$$

where $D \in \operatorname{alg} \mathcal{N}_{N}, E \in \operatorname{alg} \mathcal{N}_{N^{\perp}}$ and $F \in B\left(N^{\perp}, N\right)\left(\mathcal{N}_{N}=\{M \cap N: M \in \mathcal{N}\}\right.$ and $\mathcal{N}_{N^{\perp}}=\left\{M \cap N^{\perp}: M \in \mathcal{N}\right\}$ ). Without loss of generality, we may assume

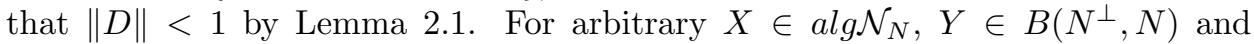
$Z \in \operatorname{alg} \mathcal{N}_{N^{\perp}}$, we write

$$
\left\{\begin{array}{l}
\varphi\left(\left[\begin{array}{cc}
X & 0 \\
0 & 0
\end{array}\right]\right)=\left[\begin{array}{cc}
A_{11}(X) & A_{12}(X) \\
0 & A_{22}(X)
\end{array}\right], \\
\varphi\left(\left[\begin{array}{ll}
0 & Y \\
0 & 0
\end{array}\right]\right)=\left[\begin{array}{cc}
B_{11}(Y) & B_{12}(Y) \\
0 & B_{22}(Y)
\end{array}\right], \\
\varphi\left(\left[\begin{array}{ll}
0 & 0 \\
0 & Z
\end{array}\right]\right)=\left[\begin{array}{cc}
C_{11}(Z) & C_{12}(Z) \\
0 & C_{22}(Z)
\end{array}\right] .
\end{array}\right.
$$


Obviously, $A_{i j}, B_{i j}$ and $C_{i j}(i, j=1,2, i \leq j)$ are linear mappings on $\operatorname{alg} \mathcal{N}_{N}$, $B\left(N^{\perp}, N\right)$ and $\operatorname{alg} \mathcal{N}_{N^{\perp}}$, respectively.

Step 1. We show that $A_{11}(\cdot)$ is a derivable mapping at $D$. For arbitrary $X, U \in$ alg $\mathcal{N}$ with $X U=D$, taking $S=\left[\begin{array}{cc}\lambda^{-1} X & \lambda E \\ 0 & \lambda F\end{array}\right], T=\left[\begin{array}{cc}\lambda U & 0 \\ 0 & \lambda^{-1} I_{N^{\perp}}\end{array}\right] \in$ alg $\mathcal{N}$ for any real number $\lambda>0$, then $S T=G$. It follows that

$$
\begin{aligned}
& {\left[\begin{array}{cc}
A_{11}(D)+B_{11}(E)+C_{11}(F) & A_{12}(D)+B_{12}(E)+C_{12}(F) \\
0 & A_{22}(D)+B_{22}(E)+C_{22}(F)
\end{array}\right] } \\
= & \varphi(G)=\varphi(S) T+S \varphi(T) \\
= & {\left[\begin{array}{cc}
\lambda^{-1} A_{11}(X)+\lambda B_{11}(E)+\lambda C_{11}(F) & * \\
0 & *
\end{array}\right]\left[\begin{array}{cc}
\lambda U & 0 \\
0 & \lambda^{-1} I_{N^{\perp}}
\end{array}\right] } \\
& +\left[\begin{array}{cc}
\lambda^{-1} X & \lambda E \\
0 & \lambda F
\end{array}\right]\left[\begin{array}{cc}
\lambda A_{11}(U)+\lambda^{-1} C_{11}(I) & * \\
0 & *
\end{array}\right] \\
= & {\left[\begin{array}{cc}
A_{11}(X) U+\lambda^{2} B_{11}(E) U+\lambda^{2} C_{11}(F) U & * \\
+X A_{11}(U)+\lambda^{-2} X C_{11}(I) & \\
0 &
\end{array}\right] . }
\end{aligned}
$$

It follows from the matrix equation that

$$
\begin{aligned}
& A_{11}(D)+B_{11}(E)+C_{11}(F) \\
& \quad=A_{11}(X) U+\lambda^{2} B_{11}(E) U+\lambda^{2} C_{11}(F) U+X A_{11}(U)+\lambda^{-2} X C_{11}(I) .
\end{aligned}
$$

The above equation implies that

$$
\begin{aligned}
A_{11}(D)+B_{11}(E)+C_{11}(F) & =A_{11}(X) U+X A_{11}(U), \\
B_{11}(E) U+C_{11}(F) U & =0, X C_{11}(I)=0 .
\end{aligned}
$$

Furthermore $C_{11}(I)=0$ and $B_{11}(E)+C_{11}(F)=0$. Thus $A_{11}(D)=A_{11}(X) U+$ $X A_{11}(U)$; i.e. $A_{11}(\cdot)$ is a derivable mapping at $D$.

Step 2. We show that $C_{11}(W)=0$ and $B_{11}(V)=0$ for any $W \in \operatorname{alg} \mathcal{N}_{N^{\perp}}$ and $V \in B\left(N^{\perp}, N\right)$.

Letting $S=\left[\begin{array}{cc}X & Y \\ 0 & Z\end{array}\right], T=\left[\begin{array}{cc}U & V \\ 0 & W\end{array}\right] \in$ alg $\mathcal{N}$ with $S T=G$, then $X U=D$, $X V+Y W=E$ and $Z W=F$. Since $\varphi$ is a derivable mapping at $G$ on algN $\mathcal{N}$, we have

$$
\begin{aligned}
& {\left[\begin{array}{cc}
A_{11}(D)+B_{11}(E)+C_{11}(F) & A_{12}(D)+B_{12}(E)+C_{12}(F) \\
0 & A_{22}(D)+B_{22}(E)+C_{22}(F)
\end{array}\right] } \\
= & \varphi(G)=\varphi(S) T+S \varphi(T) \\
= & {\left[\begin{array}{cc}
A_{11}(X)+B_{11}(Y) & A_{12}(X)+B_{12}(Y) \\
+C_{11}(Z) & +C_{12}(Z) \\
0 & A_{22}(X)+B_{22}(Y)+C_{22}(Z)
\end{array}\right]\left[\begin{array}{cc}
U & V \\
& \\
0 & W
\end{array}\right] } \\
+ & {\left[\begin{array}{cc}
X & Y \\
& \\
0 & Z
\end{array}\right]\left[\begin{array}{cc}
A_{11}(U)+B_{11}(V) & A_{12}(U)+B_{12}(V) \\
+C_{11}(W) & \\
0 & A_{22}(U)+C_{12}(W) \\
\end{array}\right] . }
\end{aligned}
$$


The above equation implies that the following three equations hold:

$$
\begin{aligned}
A_{11}(D) & +B_{11}(E)+C_{11}(F) \\
= & A_{11}(X) U+B_{11}(Y) U+C_{11}(Z) U \\
& +X A_{11}(U)+X B_{11}(V)+X C_{11}(W), \\
A_{12}(D) & +B_{12}(E)+C_{12}(F) \\
= & A_{11}(X) V+B_{11}(Y) V+C_{11}(Z) V \\
& +A_{12}(X) W+B_{12}(Y) W+C_{12}(Z) W \\
& +X A_{12}(U)+X B_{12}(V)+X C_{12}(W) \\
& +Y A_{22}(U)+Y B_{22}(V)+Y C_{22}(W), \\
A_{22}(D) & +B_{22}(E)+C_{22}(F) \\
= & A_{22}(X) W+B_{22}(Y) W+C_{22}(Z) W \\
& +Z A_{22}(U)+Z B_{22}(V)+Z C_{22}(W) .
\end{aligned}
$$

Note that $A_{11}$ is a derivable mapping at $D$. So we have $A_{11}(X U)=A_{11}(D)=$ $A_{11}(X) U+X A_{11}(U)$ for any $X, U \in \operatorname{alg} \mathcal{N}_{N}$. By (2.3), we have

$$
B_{11}(E)+C_{11}(F)=B_{11}(Y) U+C_{11}(Z) U+X B_{11}(V)+X C_{11}(W) .
$$

Taking $X=D, U=I_{N}, Y=E, V=0, Z=F$ and $W=I_{N^{\perp}}$ in (2.6), we get $D C_{11}\left(I_{N^{\perp}}\right)=0$. Letting $X=D, U=I_{N}, Y=-E, V=0, Z=-F$ and $W=-I_{N^{\perp}}$ in $(2.6), B_{11}(E)+C_{11}(F)=0$. It follows that

$$
0=B_{11}(Y) U+C_{11}(Z) U+X B_{11}(V)+X C_{11}(W) .
$$

For arbitrary $V \in B\left(N^{\perp}, N\right)$, if we put $X=I_{N}, U=D, W=I_{N^{\perp}}, Z=F$ and $Y=E-V$ in the above equation, then $B_{11}(V)(I-D)=0$. Since $\|D\|<1$ imply that $I-D$ is invertible, $B_{11}(V)=0$. For arbitrary $W \in \operatorname{alg} \mathcal{N}_{N^{\perp}}$, if we put $X=I_{N}, Y=0, Z=F W^{-1}, U=D$ and $V=E$ in the above equation, then

$$
C_{11}\left(F W^{-1}\right) D+C_{11}(W)=0 .
$$

Replacing $W$ by $2 W$ in the above equation, we have

$$
\frac{1}{2} C_{11}\left(F W^{-1}\right) D+2 C_{11}(W)=0 .
$$

The above two equations imply that $C_{11}(W)=0$ for any invertible operator $W \in$ $\operatorname{alg} \mathcal{N}_{N^{\perp}}$. By Lemma 2.2, $C_{11}(W) \equiv 0$.

Step 3. We show that $A_{22}(X)=0$ and $B_{22}(Y)=0$ for any $X \in \operatorname{alg} \mathcal{N}_{N}$ and $Y \in B\left(N^{\perp}, N\right)$.

Letting $X=D, U=I_{N}, Y=E, V=0, Z=F$ and $W=I_{N^{\perp}}$ in (2.5),

$$
F\left(A_{22}\left(I_{N}\right)+C_{22}\left(I_{N^{\perp}}\right)\right)=0 .
$$


On the other hand, for arbitrary $Y \in B\left(N^{\perp}, N\right)$ and real number $\lambda \neq 0$, putting $X=\lambda I_{N}, U=\lambda^{-1} D, V=Y+\lambda^{-1} E, Z=-\lambda^{-1} F$ and $W=-\lambda I_{N^{\perp}}$ in (2.5), we get

$$
\begin{aligned}
A_{22}(D)+B_{22}(E)= & -\lambda^{2} A_{22}\left(I_{N}\right)-\lambda B_{22}(Y)-\lambda^{-2} F A_{22}(D) \\
& -\lambda^{-1} F B_{22}(Y)-\lambda^{-2} F B_{22}(E)+F C_{22}\left(I_{N^{\perp}}\right) .
\end{aligned}
$$

The above equation implies that

$$
\begin{aligned}
& A_{22}(D)+B_{22}(E)=F C_{22}\left(I_{N^{\perp}}\right), A_{22}\left(I_{N}\right)=0, \\
& F A_{22}(D)+F B_{22}(E)=0, B_{22}(Y)=0 .
\end{aligned}
$$

It follows from (2.7)-(2.9) that $\left.F C_{22}\left(I_{N^{\perp}}\right)\right)=0$ and

$$
A_{22}(D)=F C_{22}\left(I_{N^{\perp}}\right)=0 .
$$

Apply (2.9) and the above equation to (2.5) to get

$$
C_{22}(F)=A_{22}(X) W+C_{22}(Z) W+Z A_{22}(U)+Z C_{22}(W) .
$$

For any invertible operator $X \in \operatorname{alg}_{N}$, taking $U=X^{-1} D, V=0, W=I_{N^{\perp}}$, $Y=E$ and $Z=F$ in the above equation, we have

$$
A_{22}(X)+F A_{22}\left(X^{-1} D\right)=0 .
$$

Replacing $X$ by $2 X$, we have

$$
2 A_{22}(X)+\frac{1}{2} F A_{22}\left(X^{-1} D\right)=0 .
$$

The above two equations imply that $A_{22}(X)=0$ for any invertible operator $X \in$ $\operatorname{alg} \mathcal{N}_{N}$. By Lemma 2.2, $A_{22}(X) \equiv 0$.

Step 4. We show that $A_{12}(X)=-X C_{12}\left(I_{N^{\perp}}\right)$ and $C_{12}(W)=C_{12}\left(I_{N^{\perp}}\right) W$ for any $X \in \operatorname{alg} \mathcal{N}_{N}$ and $W \in \operatorname{alg} \mathcal{N}_{N^{\perp}}$. For an arbitrary invertible operator $X \in \operatorname{alg} \mathcal{N}_{N}$, taking $U=X^{-1} D, V=0, Y=\lambda E, Z=\lambda F$ and $W=\lambda^{-1} I_{N \perp}$ in (2.4), we get

$$
\begin{aligned}
A_{12}(D)= & \lambda^{-1} A_{12}(X)+X A_{12}\left(X^{-1} D\right) \\
& +\lambda^{-1} X C_{12}\left(I_{N^{\perp}}\right)+\lambda E A_{22}\left(X^{-1} D\right)+E C_{22}\left(I_{N^{\perp}}\right) .
\end{aligned}
$$

Equation (2.10) implies that

$$
A_{12}(X)=-X C_{12}\left(I_{N^{\perp}}\right) .
$$

It follows from Lemma 2.2 that $A_{12}(X)=-X C_{12}\left(I_{N^{\perp}}\right)$ for any $X \in \operatorname{alg} \mathcal{N}$.

For an arbitrary invertible operator $W \in$ alg $\mathcal{N}_{N^{\perp}}$, taking $X=I_{N}, U=D$, $Y=0, V=E$ and $Z=F W^{-1}$ in $(2.4)$,

$$
C_{12}(F)=A_{11}\left(I_{N}\right) E+A_{12}\left(I_{N}\right) W+C_{12}\left(F W^{-1}\right) W+C_{12}(W) .
$$

Replacing $W$ by $2 W$ in the above equation, we have

$$
C_{12}(F)=A_{11}\left(I_{N}\right) E+2 A_{12}\left(I_{N}\right) W+C_{12}\left(F W^{-1}\right) W+2 C_{12}(W) .
$$

Combining (2.11) with (2.12), we obtain

$$
A_{12}\left(I_{N}\right) W+C_{12}(W)=0 .
$$


Letting $W=I_{N^{\perp}}$ in the above equation, we get $A_{12}\left(I_{N}\right)=-C_{12}\left(I_{N^{\perp}}\right)$. Hence $C_{12}(W)=C_{12}\left(I_{N^{\perp}}\right) W$. It follows from Lemma 2.2 that $C_{12}(W)=C_{12}\left(I_{N^{\perp}}\right) W$ for any $W \in \operatorname{alg} \mathcal{N}^{\perp}$.

Step 5. We show that both $A_{11}\left(I_{N}\right)=0$ and $C_{22}\left(I_{N^{\perp}}\right)=0$.

For arbitrary $V \in B\left(N^{-1}, N\right)$, taking $X=I_{N}, U=D, Y=E-V, Z=F$ and $W=I_{N^{\perp}}$ in (2.4), by the results of Steps 2 and 3, we have

$$
A_{11}\left(I_{N}\right) V=V C_{22}\left(I_{N^{\perp}}\right)-E C_{22}\left(I_{N^{\perp}}\right) \text {. }
$$

Letting $V=0$ in (2.13), we get $E C_{22}\left(I_{N^{\perp}}\right)=0$. Hence $A_{11}\left(I_{N}\right) V=V C_{22}\left(I_{N^{\perp}}\right)$ for any $V \in B\left(N^{\perp}, N\right)$. For arbitrary $x \in N$ and $y \in N^{-1}$, we have $x \otimes y \in B\left(N^{\perp}, N\right)$. So $A_{11}\left(I_{N}\right) x \otimes y=x \otimes C_{22}\left(I_{N^{\perp}}\right)^{*} y$. Thus there exists a complex number $\alpha$ such that $A_{11}\left(I_{N}\right)=\alpha I_{N}$ and $C_{22}\left(I_{N^{\perp}}\right)=\alpha I_{N^{\perp}}$. Note that $F C_{22}\left(I_{N^{\perp}}\right)=0$ and $E C_{22}\left(I_{N^{\perp}}\right)=0$. So $\alpha F=0$ and $\alpha E=0$. On the other hand, taking $X=I_{N}$, $U=D, V=E, Y=0, Z=I$ and $W=F$ in (2.3), we get $A_{11}\left(I_{N}\right) D=0$, i.e. $\alpha D=0$. Since $G \neq 0, \alpha=0$. It follows that $A_{11}\left(I_{N}\right)=0$ and $C_{22}\left(I_{N^{\perp}}\right)=0$.

Step 6 . We show that both $A_{11}(\cdot)$ and $C_{22}(\cdot)$ are derivations.

For arbitrary $Y \in B\left(N^{\perp}, N\right)$ and invertible operator $W \in \operatorname{alg} \mathcal{N}_{N^{\perp}}$, taking $X=I_{N}, U=D, V=E-Y W$ and $Z=F W^{-1}$ in (2.4), we obtain

$$
B_{12}(Y W)=B_{12}(Y) W+Y C_{22}(W) .
$$

For any $W_{1}, W_{2} \in \operatorname{alg} \mathcal{N}_{N^{\perp}}$, we have

$$
B_{12}\left(Y W_{1} W_{2}\right)=B_{12}(Y) W_{1} W_{2}+Y C_{22}\left(W_{1} W_{2}\right)
$$

On the other hand,

$$
\begin{aligned}
B_{12}\left(Y W_{1} W_{2}\right) & =B_{12}\left(Y W_{1}\right) W_{2}+Y W_{1} C_{22}\left(W_{2}\right) \\
& =B_{12}(Y) W_{1} W_{2}+Y C_{22}\left(W_{1}\right) W_{2}+Y W_{1} C_{22}\left(W_{2}\right) .
\end{aligned}
$$

Then (2.15) and (2.16) imply that

$$
C_{22}\left(W_{1} W_{2}\right)=C_{22}\left(W_{1}\right) W_{2}+W_{1} C_{22}\left(W_{2}\right)
$$

Hence $C_{22}(\cdot)$ is a derivation.

For arbitrary $V \in B\left(N^{\perp}, N\right)$ and invertible operator $X \in \operatorname{alg} \mathcal{N}_{N}$, taking $X^{-1} D$, $Y=E-X V, W=I_{N^{\perp}}$ and $Z=F$, we obtain

$$
B_{12}(X V)=A_{11}(X) V+X B_{12}(V)
$$

Similarly, we can prove that $A_{11}(\cdot)$ is a derivation.

Step 7. We show that $\varphi$ is a derivation. For arbitrary $S=\left[\begin{array}{cc}X & Y \\ 0 & Z\end{array}\right]$ and $T=$ $\left[\begin{array}{cc}U & V \\ 0 & W\end{array}\right]$ in algN $\mathcal{N}$, we only need to prove that $\varphi(S T)=\varphi(S) T+S \varphi(T)$. By 
(2.14), (2.17) and the results of Steps 2-6, we easily calculate

$$
\begin{aligned}
& \varphi(S) T+S \varphi(T) \\
& =\left[\begin{array}{cc}
A_{11}(X) & A_{12}(X)+B_{12}(Y)+C_{12}(Z) \\
0 & C_{22}(Z)
\end{array}\right]\left[\begin{array}{cc}
U & V \\
0 & W
\end{array}\right] \\
& +\left[\begin{array}{cc}
X & Y \\
0 & Z
\end{array}\right]\left[\begin{array}{cc}
A_{11}(U) & A_{12}(U)+B_{12}(V)+C_{12}(W) \\
0 & C_{22}(W)
\end{array}\right] \\
& =\left[\begin{array}{cc}
A_{11}(X) U+X A_{11}(U) & A_{11}(X) V+A_{12}(X) W+B_{12}(Y) W \\
+C_{12}(Z) W+X A_{12}(U)+X B_{12}(V) \\
\\
0
\end{array}\right] \\
& =\left[\begin{array}{cc}
A_{11}(X U) & A_{12}(X) W+B_{12}(Y W)+C_{12}(Z) W \\
& +X A_{12}(U)+B_{12}(X V)+X C_{12}(W) \\
0 & C_{22}(Z W)
\end{array}\right] \\
& =\left[\begin{array}{cc}
A_{11}(X U) & A_{12}(X) W+B_{12}(Y W+X V) \\
& +C_{12}(Z) W+X A_{12}(U)+X C_{12}(W) \\
0 & C_{22}(Z W)
\end{array}\right] \\
& =\left[\begin{array}{cc}
A_{11}(X U) & -X C_{12}\left(I_{N^{\perp}}\right) W+B_{12}(Y W+X V) \\
& +C_{12}\left(I_{N^{\perp}}\right) Z W-X U C_{12}\left(I_{N^{\perp}}\right)+X C_{12}\left(I_{N^{\perp}}\right) W \\
0 & C_{22}(Z W)
\end{array}\right] \\
& =\left[\begin{array}{cc}
A_{11}(X U) & B_{12}(Y W+X V)+C_{12}\left(I_{N^{\perp}}\right) Z W-X U C_{12}\left(I_{N^{\perp}}\right) \\
0 & C_{22}(Z W)
\end{array}\right] \\
& =\left[\begin{array}{cc}
A_{11}(X U) & B_{12}(Y W+X V)+C_{12}(Z W)+A_{12}(X U) \\
0 & C_{22}(Z W)
\end{array}\right] \\
& =\varphi(S T) \text {. }
\end{aligned}
$$

This completes the proof.

\section{REFERENCES}

1. M. Brešar, Characterization of derivations on some normed algebras with involution, J. Algebra 152 (1992), 454-462. MR1194314(94e:46098)

2. M. Brešar, P. Šemrl, Mappings which preserve idempotents, local automorphisms, and local derivations. Canad. J. Math. 45 (1993), 483-496. MR.1222512 (94k:47054)

3. R.L. Crist, Local derivations on operator algebras, J. Funct. Anal. 135 (1996), 76-92. MR:1367625 (96m:46128)

4. K.R. Davidson, Nest algebras, Research Notes in Math. No. 191, Longman Sci. \& Tech., Wiley \& Sons, New York, 1988. MR0972978 (90f:47062)

5. J.A. Erdos, Operators of finite rank in nest algebras, J. London Math. Soc. 43 (1968), 391-397. MR0230156 (37:5721)

6. J.C. Hou, X.F. Qi, Characterizations of derivations of Banach space nest algebras: allderivable points, Linear Algebra Appl. 432 (2010), 3183-3200. MR.2639278 (2011j:47114)

7. W. Jing, S.J. Lu, P.T. Li, Characterizations of derivations on some operator algebras, Bull. Austral. Math. Soc. 66 (2002), 227-232. MR1932346 (2003f:47059) 
8. R.V. Kadison, Local derivations, J. Algebra 130 (1990), 494-509. MR1051316 (91f:46092)

9. D.R. Larson, A.R. Sourour, Local derivations and local automorphisms of $\mathcal{B}(X)$, Operator Algebras and Applications, Proc. Symp. Pure Math. 51, Amer. Math. Soc., 1990, 187-194. MR1077437 (91k:47106)

10. D.R. Larson, Nest algebras and similarity transformations, Annals of Mathematics 121 (1985), 409-427. MR0794368 (86j:47061)

11. J.K. Li, Z.D. Pan, H. Xu, Characterizations of isomorphisms and derivations of some algebras. J. Math. Anal. Appl. 332 (2007), 1314-1322. MR2324339 (2008e:47093)

12. P. Šmrl, Local automorphisms and derivations on $B(H)$, Proc. Amer. Math. Soc. 125 (1997), 2677-2680. MR 1415338 (98e:46082)

13. J. Zhu, All-derivable points of operator algebras. Linear Algebra Appl. 427 (2007), 1-5. MR2353150 (2008g:47141)

14. J. Zhu, C.P. Xiong, Derivable mappings at unit operator on nest algebras. Linear Algebra Appl. 422 (2007), 721-735. MR2305152(2008a:47123)

15. J. Zhu, C.P. Xiong, Bilocal derivations of standard operator algebras, Proc. Amer. Math. Soc. 125 (1997), 1367-1370. MR1363442 (97g:46091)

16. J. Zhu, C.P. Xiong, R.Y. Zhang, All-derivable points in the algebra of all upper triangular matrices. Linear Algebra Appl. 429(4) (2008), 804-818. MR2428131 (2009m:47200)

Institute of Mathematics, Hangzhou Dianzi University, Hangzhou 310018, People's Republic of China

E-mail address: junzhu@yahoo.cn

Institute of Mathematics, Hangzhou Dianzi University, Hangzhou 310018, People's Republic of China 